International Journal of Electrical Engineering and Technology (IJEET)

Volume 11, Issue 4, June 2020, pp. 135-140, Article ID: IJEET_11_04_015

Available online at https://iaeme.com/Home/issue/IJEET? Volume $=11 \&$ Issue $=4$

ISSN Print: 0976-6545 and ISSN Online: 0976-6553

DOI: https://doi.org/10.34218/IJEET.11.4.2020.015

(C) IAEME Publication

Scopus Indexed

\title{
LAYOUT VERIFICATION OF HARD MACROS
}

\author{
Natesh Gowda G M \\ Department of Electronics and Communication Engineering, \\ RV College of Engineering, Bengaluru, India \\ Sujatha Hiremath \\ Department of Electronics and Communication Engineering, \\ RV College of Engineering, Bengaluru, India
}

\begin{abstract}
Design of modern day Integrated Circuits design process is split up into Front end design and back end design or Physical Design. Physical design includes many steps like partitioning, floor planning, Placement, clock tree synthesis, signal routing and timing closure. As elevation in area utilization, complexity in design and decrease in technology node, Placement and Routing tools are not able to provide Timing, LVS and DRC clean database. Layout verification is a check to address the violations and provides physically clean Graphic Database system to foundary to manufacture. Layout verification includes many subchecks which include Design Rule Check, Density check, Antenna check, Electric rule check, Layout vs Schematic check. This paper presents the detailed steps in Physical design and role of Layout verification in each steps

Key words: Electric Design Automation, Design Rule Check, Electrical Rule Check, Intellectual Property, Placement and Routing, Base Tape out, Metal Tape Out

Cite this Article: Natesh Gowda G M and Sujatha Hiremath, Layout Verification of Hard Macros, International Journal of Electrical Engineering and Technology, 11(4), 2020, pp. 135-140.

https://iaeme.com/Home/issue/IJEET?Volume=11\&Issue $=4$
\end{abstract}

\section{NTRODUCTION}

Advanced mobile platforms includes many subsystems like Audio, Video, Modem, Peripherals, Memories and Analog blocks. As standard of Video, Camera, Performance, Connectivity expanding, the complexity of the subsystems also increases. As the functionality of subsystems increases, logic cells need to perform these operation also increases. This complexity makes the Placement and routing tools to fails in generating error free layouts. Layout verification is a process where an integrated circuit physical Layout is validate using sign off tools like calibre to secure Physically, Electrically, Logically and Manufacturable Design. Layout verification includes Design Rule check which ensure that design is still functional even when there may be lots of misalignment and various side effects during 
manufacturing process. Density rule ensures the even density throughout the chip, Layout vs Schematic ensures physical Layout corresponds to original circuit diagram or schematic of the design. The rest of the paper is a follows: Section II presents Literature Review, which discuss existing Layout checks. Section III discuss Methodology of Layout Verification, Section IV describes Layout Verification Nodes. Section V describes Layout verification checks and Section VI discuss the example of Automation and SectionVII concludes the paper.

\section{LITERATURE REVIEW}

Due to high complexity and decrease in technology node, Layout that designed consists of many errors that needs to be verify, this creates the bridging gap between physical design and foundry fabrication. The Literature review reflects the development in the physical design and layout verification methodology over the years. Challenges and Implementation of latest process technology for the designers from foundry's perspective requires innovation in EDA tools [1]. Design rules specified from the industry requires comfortable user interface which requires efficient use of computer resources[2]. New technology trends and their impact on partitioning, floor planning, placement and routing are discussed in [3].[4,8] presents automation of Electric Rule check, Design Rule check, Layout vs schematic check. The important factor which determines the reliability and high yield is the Electrostatic discharge, ESD protection for design for manufacturing is discussed in [9].Antenna effect or In process effect determines the reliability and yield in nano meter era [10] discuss the discharge path based In process detection method.

\section{METHODOLOGY}

Flow diagram in the below Fig. 1 shows the methodology incorporated to verify layout in IC manufacturing process. This section describes the implementation method from floorplan till the design reach foundary.

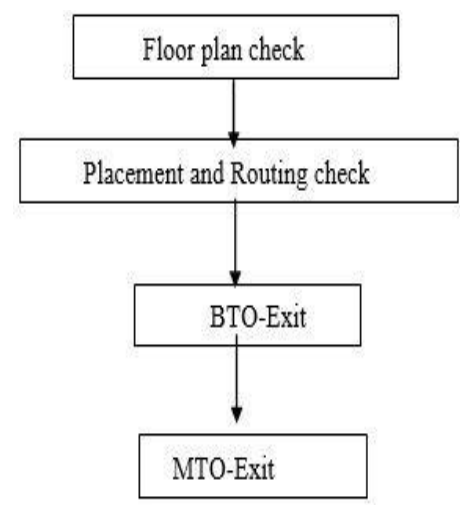

Figure 1 Methodology of Layout Verification

At floor-plan check stage all pre-placed cells ,IPs, Memories, Tap cells, Tie Cells, Decap Cells and Power distribution design are placed. Performing Base DRC at this stage to correct any miss orientation in preplaced cells and to check the presence of tie cells at specific interval and Base Density. Execution of Softcheck and Electric Rule Check at this stage to removes any short between different power and Power and Ground Opens in the design respectively Performing ESD check to verify the presence of different ESD clamp Cells at corresponding power domain . At Placement and Routing check stage, placement of logic cells and their interconnections, clock network and secondary power distribution are placed Carry out Metal DRC and Density checks to removes the Metal DRC and Density issues in the design Performing Softcheck to find any nwell/psub power connections, secondary power 
shorts. Carry out Antenna check verifies the antenna ratio at each gate Performing Layout vs Schematic to verifies the functionality of the layout with respect to original circuit topology Performing Metal DRCs at BTO and MTO stages to check DRCs which causes due to addition of timing ECOs in the design

\section{LAYOUT VERIFICATION NODES}

Layout verification consists of many check to be performed before hand off data to the foundary. Fig. 2 shows the layout verification node that automatically generates the data required for the signoff tool to check correctness of the design.

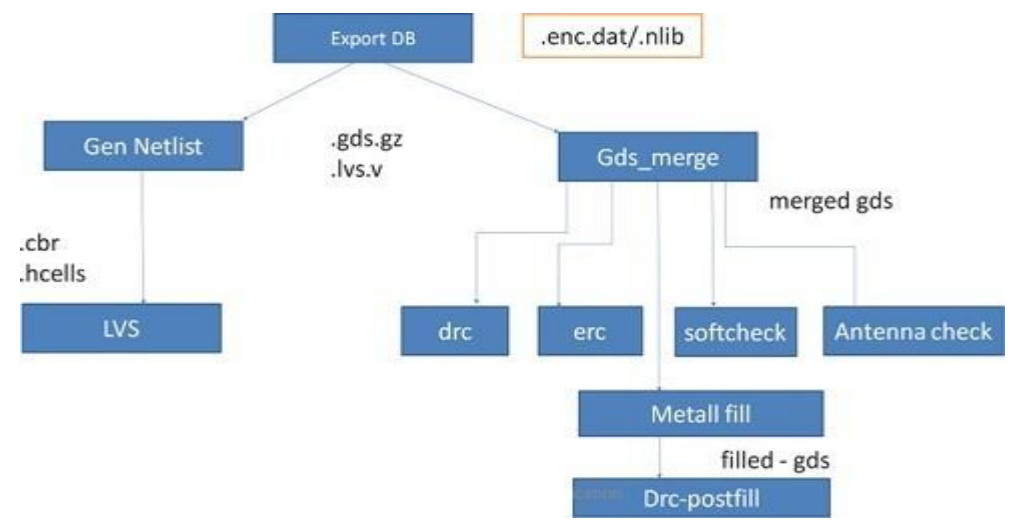

Figure 2 Layout Verification Nodes

Export database is the process which dumps the information like left information of the cell, gets format of pnr data and the netlist required for the LVS. Netlist (.cbr) is extracted from lvs.v which is used to perform Layout vs Schematic . gets merging is a process where all the base layer information of memories ,IPs ,Logic cells are added from cell library Merged GDS is used to check DRC, Softcheck, ERC, Antenna checks .MFILL is the process where dummy layers are added in the free space to maintain even density across chip. This filled GDS is used to perform DRC post fill and density checks.

\section{LAYOUT VERIFICATION CHECKS}

Many layout checks are performed to ensure faultless data to be deliver for the foundary, these includes Design Rule Check, Antenna check, Density check, Electric rule check, Softcheck, Layout vs Schematic. Below section discuss the functionality and input files required for each checks to run.

\subsection{Design Rule Check}

Design Rule check is performed to verify constancy and to improve the gross yield in the database. All the design rules are technology and foundary dependent. All the design rules are embedded in rule deck file and provide as input to signoff tool. Input files includes database in the form of gds and run set files. Design rule check is classified as Base DRC and Metal DRC. Base DRC mainly checks for FEOL layers such as Diffusion, Poly and Poly contact. Metal DRC checks for BEOL layers which interconnects the Transistor.

\subsection{Antenna Check}

Antenna or in process check required to verify the allowable ratio of matal area to gate area at each transistor. This failure mainly takes place during manufacturing process which causes accumulation of charges on the metal and discharges to gate through gate oxide which causes 
damage to transistor. Main fixes for antenna violation is add diode to discharge of extra electrons or to change the order of routing.

\subsection{Electric Rule Check}

Electric rule check is used to verify the diffusion, substrate and gate connectivity in the design. Some of the connections like nwell to power, substrate to ground is verified in Electric rule check. Input files required are Layout data in the form of gds or aosis, run set file, different power and ground definitions, edtext file.

\subsection{Softcheck}

Softcheck is used to check the presence of merged domain cells in non merged domain and also to check the different power or power ground physical shorts Input files required are layout files in the for of gds or oasis, runset file, power and ground definitions, edtext file.

\subsection{Layout vs Schematic}

Design rule check (DRC) ensures that the layout follows the rules required for faultless fabrication, but not guarantees if it really represents the circuit desire to fabrication, for this Layout vs schematic is used to check. Layout vs Schematic consists of mainly three steps which includes Extraction, Reduction and Comparison. During extraction phase information like transistor connectivity, interconnection, cell count, power connectivity is extracted and reduced into ASCII format during reduction phase and compared with the golden netlist extracted from the circuit or schematic.

\subsection{Density Check}

Density check is performed to check the even density through out the chip which required for manufacturing process to ensure the mechanical sturdiness of the chip to achieve planarity during CMP (Chemical Mechanical Polishing). Different density checks verify the overall density of each metal and densities per unit area. In density checking, a window of predefined area is stepped, with a pre-defined step size, across a design or region.

\section{AUTOMATION OF DRC}

Every operations in the Placement and routing tool is done through commands running in the background of the tool. Using these commands as a scripts we can address DRCs in the design. Algorithms for automation of spacing DRCs is explained in this section.
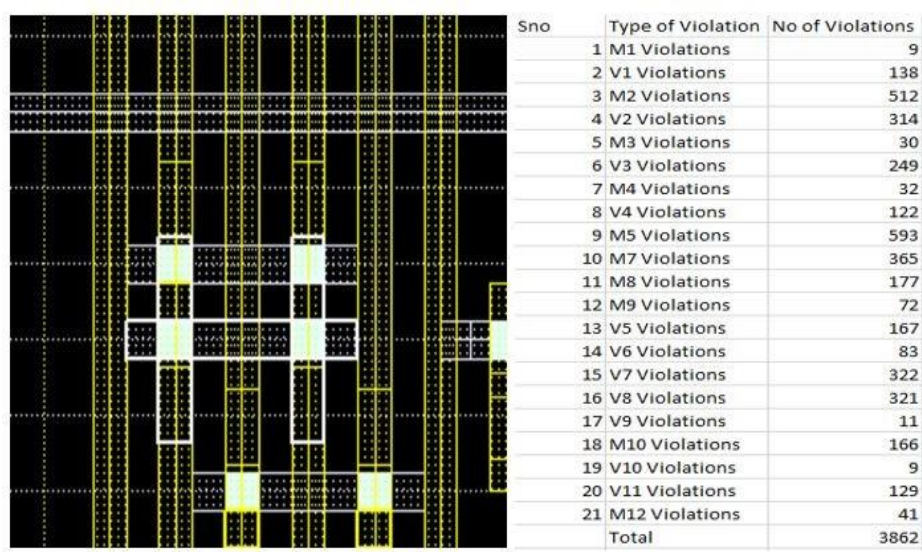

Figure 3 Pre Automation Design and DRC results 
In the above power connection tool is placing extra pair of vias on the metal causes spacing issue between the vias, as shown in the above summary file in the Fig. 3 of the DRC, nearly 322 via DRC are due to this V7 via of the same pattern which is the long process to select and delete via at each place in the design. since the pattern of the DRC is same we can address this type of DRC by writing scripts in tcl.

Algorithm for select and delete vias over the power nets as shown in Fig .4, it creates the area to search the particular via in the design

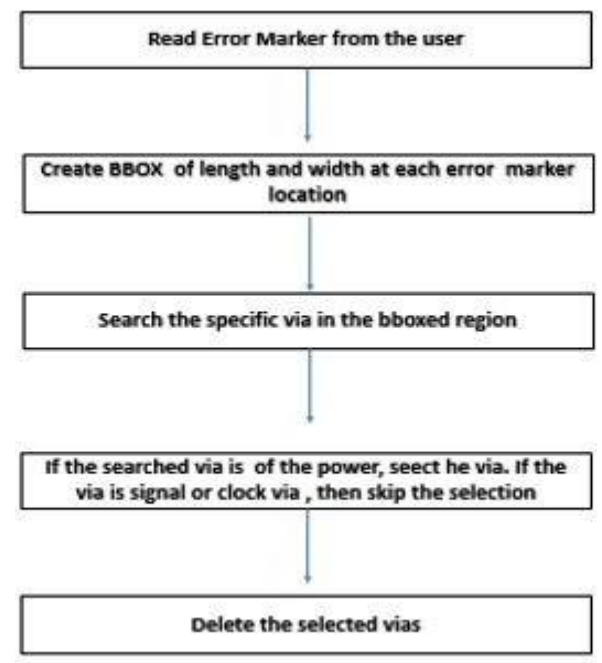

Figure 4 Algorithm of automation

- Sign off tools like calibre provides error markers using run set file and gds data in the format of .err .this error marker is updated in the Placement and routing tools like ICC or Innovus using read drc command.

- Since error marker contains the location of the error, Since searching of particular layer occurs through area, we should define bbox by using create bbox command

- After creating the box, tool need to search v7 via over the particular boxed region using search command

- If the searched via is of signal via, need to ignore. If the searched via is of power via, select the via using change selection command

- After selecting the power via, need to delete the selected via using edit select delete command

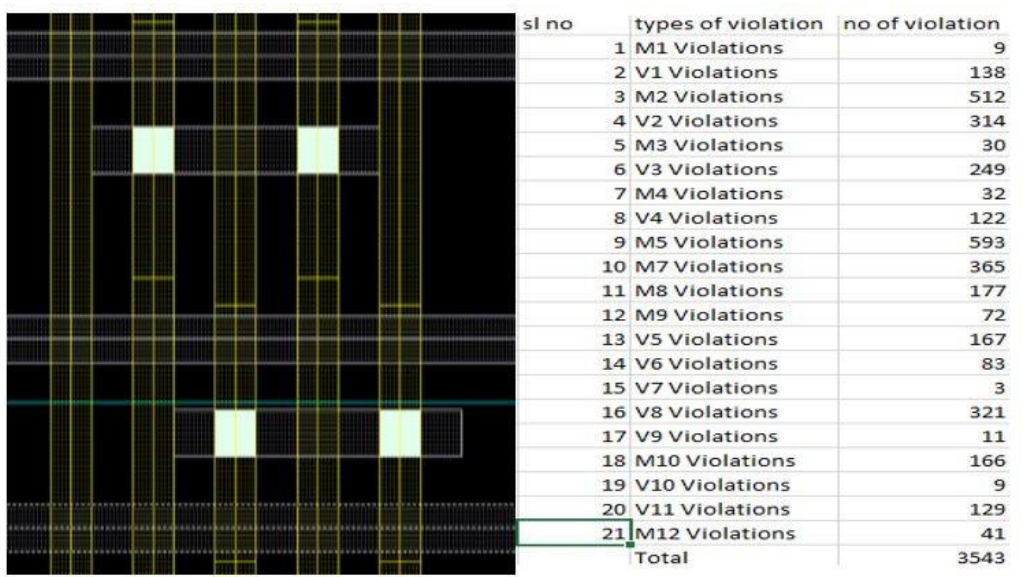

Figure 5 Post Automation Design and DRC results 
Fig. 5 shows the post sourcing of tcl in the ICC or Innovus shell, from the summary file we can conclude that the DRCs are reduced from 322 DRCs to 3 DRCs .Extra vias in the design got deleted in the design

\section{CONCLUSIONS}

As technology node decreases, number of design rules and logic cells incorporated in the advanced mobile platforms increases. The explained methodology of Layout verification at each phase of design ensures no congestion at Base Tape- out and Metal tapeout time which results in faster time to tapeout. Checking proper orientation of the cells at early stage of the design ensures no changes in floor plan at later stage of the design. Electric rule check and softcheck is the part of LVS check which makes debugging of LVS is easier and also to detect the power shorts in the mesh. This method- ology is incorporated in many industries to design mobile platforms. Automation of DRCs during BTO and MTO stages helps in decreasing manual effort as well as faster time to tape out.

\section{REFERENCES}

[1] H. S. Lee, (2015)"IC design challenges and opportunities for advanced process technology," VLSI Design, Automation and Test (VLSI-DAT), Hsinchu, pp. 1-2. doi: 10.1109/VLSI-DAT.2015.7114549.

[2] W. Ries, (1989) "Rule-based implementation of correct and efficient VLSI design rule checking," International Workshop on Industrial Applications of Machine Intelligence and Vision,, Roppongi, Tokyo, Japan, pp. 205-209.

[3] S. Sur-Kolay, P. Dasgupta, B. B. Bhattacharya and S. T. Zachariah, (2004)"'Physical design trends and layout-based fault modeling," 17th International Conference on VLSI Design. Proceedings, Mumbai, India, pp. 6-8.

[4] B. Baidya and T. Mukherjee, (2005) "Layout verification for mixed-domain integrated MEMS," in IEEE Transactions on Computer-Aided Design of Integrated Circuits and Systems, vol. 24, no. 4, pp. 563-577.

[5] R. Fischbach, A. Heinig and P. Schneider, (2014) "Design rule check and layout versus schematic for 3D integration and advanced packaging," 2014 International 3D Systems Integration Conference (3DIC), Kinsdale, pp. 1-7.doi: 10.1109/3DIC.2014.7152150.

[6] A. Arafa et al., (2012) "Schematic-driven physical verification: Fully automated solution for analog IC design," 2012 IEEE International SOC Conference, Niagara Falls, NY, pp. 260-264.doi: 10.1109/SOCC.2012.6398358.

[7] V. Immaneni and D. Puffer, (1989)"Electrical design rule checker for core and block based ASIC designs," Proceedings, Second Annual IEEE ASIC Seminar and Exhibit,, Rochester, NY, USA, pp. P6/2/1-P6/2/4. doi:10.1109/ASIC.1989.123204

[8] R. M. C. Roberts and C. J. Fourie, (2015) "Layout-Versus-Schematic Verification for Superconductive Integrated Circuits," in IEEE Transactions on Applied Superconductivity, vol. 25, no. 3, pp. 1-5, June, Art no.1200105. doi: 10.1109/TASC.2014.2373035

[9] Q. Li, Y. J. Huh, J. W. Chen, P. Bendix and S. M. Kang, (2001) "Full chip ESD design rule checking," ISCAS 2001. The 2001 IEEE International Symposium on Circuits and Systems (Cat. No.01CH37196), Sydney, NSW, pp. 503-506 vol. 5.doi: 10.1109/ISCAS.2001.922095

[10] G. Debnath and P. Thadikaran, (2009)"Design for Manufacturability and Reliability in Nano Era," 2009 22nd International Conference on VLSI De- sign, New Delhi, pp. 3334.doi: 10.1109/VLSI.Design.2009.115. 